\title{
La satisfacción laboral y la satisfacción del cliente en la atención brindada en el Club Departamental Arequipa
}

\author{
Work satisfaction and customer satisfaction in the atention \\ provide for Arequipa Departamental Club
}

Fernando Pino Apablaza $\square$

Universidad San Martín de Porres. Lima, Perú

Recibido: 30/10/2020

Revisado: $12 / 11 / 2020$

Aceptado: 20/11/2020

Publicado: 16/12/2020

\section{RESUMEN}

La investigación determinó la influencia entre los niveles de satisfacción laboral de los trabajadores del Club Departamental Arequipa en los niveles de satisfacción de los clientes, con respecto a la calidad del servicio. La investigación inició en el primer trimestre del 2018, y concluyó a fines del segundo trimestre del mismo año en el distrito de Jesús María, en la provincia de Lima. La investigación es de tipo correlacional, y para medir la satisfacción de ambos grupos se utilizaron dos instrumentos de acopio de datos tipo Likert. Para probar la hipótesis, se utilizó la prueba inversa, utilizando el coeficiente de correlación de Pearson y la prueba de análisis de la varianza Anova. Tras la prueba de hipótesis, se rechazó la hipótesis nula y se comprobó hasta que se demuestre lo contrario, la existencia de una relación positiva entre los niveles de satisfacción de los trabajadores del club en los niveles de satisfacción de los clientes, y además de una diferencia significativa entre las medias estadísticas de ambos grupos, antes y después de la aplicación de un programa de mejora de la calidad de vida laboral. Palabras clave: Satisfacción laboral, satisfacción del cliente, calidad del servicio, calidad de vida laboral.

\section{ABSTRACT}

The investigation determined the influence between the labor satisfaction levels and the satisfaction customers levels, in relation to quality of services provides for the organization. The investigation started in the first trimester of 2018 and finished the second trimester in the same year in district of Jesus Maria, province of Lima. The kind of investigation is correlation. For measure the satisfaction levels in both groups, was used two instruments to collect data. In the hypothesis proof, was used the inverse proof, using the Pearson coefficient and the Anova to analyze the difference between variances. Finally the null hypothesis was rejected and prove the existence of the positive relation between the satisfaction levels of employees and customers. Also evidenced a significant diferences between the statistics medias in both groups, after and before to the application of improvement program about the life worker quality. Keyswords: Labor satisfaction, customer satisfaction, services quality, life worker quality. 


\section{INTRODUCCIÓN}

Gran problema para las organizaciones que reciben visitantes nacionales y extranjeros como es el caso del Club Departamental Arequipa, es tener trabajadores insatisfechos. Los trabajadores insatisfechos ejecutan mal sus procesos, ya sean estos de producción o de servicio. Además, es muy posible que maltraten a los clientes y como corolario también hay que decir que son grandes detractores de la organización. Por lo tanto, la insatisfacción laboral debe ser inmediatamente atacada, pero hay que advertir que no es cosa fácil de resolver. Lo primero que se debe de hacer es medir los niveles de satisfacción de los trabajadores, considerando múltiples aspectos como lo son: El comportamiento de los administradores, el contexto organizacional, el diseño de puestos y sus diferencias individuales. Después de la medición y respectiva ponderación, los directores de negocios recién obtendrán una visión real sobre qué tan satisfechos o insatisfechos se encuentran los trabajadores de su organización. Otro punto importante a tratar, es sobre la influencia que genera la satisfacción de los trabajadores en los niveles de satisfacción de los clientes. La mayoría de directores responsables de organizaciones, solo se preocupan solamente por la satisfacción de los clientes y eso es comprensible, porque el cliente con su dinero incrementa la rentabilidad de la organización, pero no se deben de olvidar de la satisfacción de los empleados, que en gran parte es factor y causa de la satisfacción de los clientes. Bajo esa premisa, es que se realizó esta investigación, con la finalidad de demostrar la influencia que tiene la satisfacción de los trabajadores en los niveles de satisfacción de los clientes del Club Departamental Arequipa.

\section{El interés por la calidad}

Para (Parra López \& Calero García, 2006) la calidad es: "Un concepto cuya definición puede entrañar una enorme dificultad. Este concepto es por naturaleza abstracto, difícil de tangibilizar y además se ve afectado por una gran cantidad de factores subjetivos" (p.198). Según (Planells \& Montserrat, 2012) afirman que: "La calidad, viene definida por el cliente. Es el cliente el que decide si un servicio es de calidad o no" (p.276). Pero las reflexiones sobre la calidad devienen de muchos siglos atrás. El origen de la palabra calidad proviene de la antigua Grecia, del término poiotes, que significa: de qué clase, este término inventado por Platón en el siglo IV antes de Cristo, apareció por primera vez en su obra Teeteto. Posteriormente, es Aristóteles quien introduce en su obra titulada Metafísica el termino poiotes, entendido como cualidad. Posteriormente, Marco Tulio Cicerón el jurista romano en el siglo I antes de Cristo, la traduce al latín como qualitas, término que es parecido a la palabra inglesa quality, que significa calidad. En el presente la calidad sigue siendo tema importante de reflexión en varios ámbitos, como por ejemplo en el sector turístico, donde existe un Código Ético Mundial para el Turismo, elaborado por la Organización Mundial del Turismo (OMT), que en su artículo seis, exhorta imperativamente a las instituciones turísticas a brindar calidad en sus productos y servicios.

En este mismo sentido (Setó Pamies, 2004) afirma que: "no es suficiente con reconocer que la calidad es importante, sino que es necesario realizar los esfuerzos suficientes para poder convertirla en una fuente de ventaja competitiva (p.6). Y estos esfuerzos se pueden traducir en realizar investigaciones para determinar cuáles son las reales percepciones y necesidades de los clientes. En este mismo sentido (García Buades, 2001) afirma que: "En la última década se ha producido un aumento 
generalizado por el interés por la calidad, tanto en organizaciones industriales como organizaciones de servicios" (p.13). Entonces, que las organizaciones muestren interés es normal porque la calidad traerá consigo múltiples beneficios. Para (Gasalla, 2009): "La calidad siempre es rentable para la empresa (p. 201). En este mismo sentido (Zeithaml, Parasuraman, \& Berry, 1993) afirman que: "Los líderes del servicio, creen fundamentalmente que la alta calidad produce resultados positivos en la línea final de sus estados financieros" (p.9).

Además, (Hoffman \& Bateson, 2012) afirman que: "De hecho satisfacer y superar las expectativas de los clientes puede redituar en beneficios valiosos para la empresa" (p. 290). Entonces, perseguir la calidad es una excelente opción, pero como cuesta conseguirla. Sin embargo, se sabe que este, es el mejor camino a seguir. En este mismo sentido (Acerenza, 2004) afirma que: "La calidad constituye uno de los factores clave del éxito más importante de la gestión de marketing de servicios turísticos, por cuanto fortalece la competitividad [...] y contribuye al mejoramiento de los resultados económicos" (p.63).

\section{La calidad para la supervivencia organizacional}

Las empresas turísticas tienen que hacer denotados esfuerzos para sobrevivir. Como entes orgánicos tienen un ciclo de vida, análogamente similar al ciclo de vida de los seres humanos. Así es que las empresas turísticas tienen etapas correspondientes al ciclo de vida, donde inicia con un nacimiento, una etapa de juventud, madurez, y muerte. Las empresas turísticas para no morir prematuramente tienen que hacer grandes esfuerzos en un ámbito turístico competitivo, donde la competencia es agresiva, perniciosa y mortal. Por lo tanto, las empresas turísticas tienen que cuidarse de la competencia y además, entender cómo es el entorno donde se encuentran circunscritas. Pero, también tienen que entender que la calidad es el camino al éxito empresarial. En este mismo sentido (Setó Pamies, 2004) afirma que: "La calidad es considerada, tanto por las empresas industriales y de servicios, como un arma estratégica para poder sobrevivir y especialmente en entornos altamente competitivos" (p.6).

(Fernández \& Bajac, 2012)Afirma que: "Muchas firmas aducen diferenciarse por la calidad de su servicio. Los empresarios saben que un servicio de alta calidad puede representar una oportunidad de negocio, o la diferencia entre la continuidad y la desaparición de una marca" (p. 346). También (Olvera Romero, 2013) menciona sobre la calidad de servicio y la supervivencia de las organizaciones, que:

Las empresas viven de las ventas y esto implica que deberán cuidar que lo que se venda tenga una calidad adecuada y que los servicios que se ofrezcan estén dirigidos a las necesidades y gustos de los clientes; de lo contrario, quebrarían, ya que los clientes preferirán a la competencia (p.15).

En este mismo sentido (Couillaud, 2006) afirma que: "Una empresa sin clientes no puede subsistir; y que el personal de la empresa debe realizar su labor de tal forma que el consumidor esté plenamente a gusto con ella" (p.48).

Y en el sentido laboral también ocurre lo mismo. Según Baguer como se citó en (Arbaiza Fermini, 2014), menciona que el personal debe tener las siguientes características: "Las personas deben usar su creatividad en el diseño y la planificación, y estar motivadas para alcan- 
zar las metas; en caso contrario, la empresa es incapaz de sobrevivir al ambiente" (p.239).

\section{La calidad de servicio}

Las empresas efectivas, luchan día a día por brindar calidad en sus servicios. Saben que la calidad es fuente de ventaja competitiva ante los adversarios que posiblemente tengan el mismo afán por la búsqueda de la calidad en los servicios. Según (Cobra, 2000): "La calidad es un elemento importante para definir un servicio ofrecido ya que, con base a este criterio, el consumidor establece la diferencia entre el servicio de una empresa y el de sus competidores" (p. 140). Respecto a las dimensiones de los servicios de calidad (Martin, 1992) afirma que:

Los servicios de calidad constan de dos dimensiones integrales. Una es la dimensión de los procedimientos. Esta dimensión de los servicios es sistémica por naturaleza. Trata de los sistemas de prestaciones de los servicios e incluye los procedimientos de cómo hacer las cosas. También provee los mecanismos para hacerle frente a las necesidades de los clientes. La otra es la dimensión personal. Mientras que la dimensión de los procedimientos es fría, racional y sistémica, está es cálida, con frecuencia irracional y ciertamente imposible de predecir. Este es el aspecto humano de los servicios, interpersonal por naturaleza. Comprende las actitudes, conductas y destrezas verbales que siempre están presentes en las interacciones de servicios personales (p.20).

\section{La satisfacción del cliente}

Según los expertos en marketing (Kotler \& Armstrong, 2008) afirman que la satisfacción es: "El nivel del estado de ánimo de una persona que resulta de comparar el rendimiento percibido de un producto o servicios con sus expectativas" (p.40). La satisfacción del cliente es un término que se estudia con mucho interés en el ámbito de los negocios. Según (Elias, 2000) afirma que:

La satisfacción es el estado psicológico positivo de un cliente en relación con una empresa. Insatisfacción viene a ser (visto como diferencial semántico) lo que definiría el estado contrapuesto, el psicológico negativo. La Satisfacción es el final de un proceso y como tal, tiene que ver con la experiencia del cliente en relación con sus expectativas. Al ser un estado emocional, La satisfacción debe ser medida para poder establecer una relación directa con los resultados de la empresa y de esta forma saber qué tipo de "utilidad” se está dando a los clientes (p. 16).

Según (Grande Esteban, 2005) afirma sobre la satisfacción del consumidor que: "Es el resultado de comparar su percepción de los beneficios que obtiene con las expectativas que tenía de recibirlos" (p. 345). En este mismo sentido (Chiavenato, 2011), afirma que: “El cliente debe ser la persona más importante de la organización. Jamás debe ser tratado como inoportuno o como receptor pasivo de los productos o servicios de la organización". ¿Qué sería de una organización sin clientes? (p.434).

\section{La calidad del servicio y la satisfacción del cliente}

Las organizaciones turísticas deben imperativamente ser cuidadosas a la hora de ofrecer sus servicios a los clientes, ya que, de la calidad de los servicios, dependerá en gran parte la satisfacción del cliente. En este mismo sentido (Goeldner \& Brent Ritchie, 2011) afirman 
que: "Los sectores de operación del turismo son responsables de proporcionar experiencias memorables y de alta calidad" (p.18). Y son los trabajadores quienes brindan los servicios y naturalmente son los clientes los que percibirán si los servicios fueron de calidad o no. Sí los clientes percibieron que los servicios fueron de calidad, eso quiere decir que la empresa está haciendo bien las cosas, pero si los clientes perciben que los servicios fueron de poca calidad, eso significa problemas para la organización, que deberán ser resueltos con premura, considerando que la calidad del servicio es eje fundamental para lograr la satisfacción del cliente. Además, aquellas organizaciones que no se preocupen por la calidad de sus servicios estarán en desventaja en relación a las demás organizaciones que sí se preocupan. En este mismo sentido (Setó Pamies, 2004) afirma que:

En el entorno actual la calidad de servicio se convierte en una de las variables consideradas clave para la competitividad de la empresa. Las empresas tienen claro que, si quieren sobrevivir en un mercado tan competitivo como el actual, ofrecer una elevada calidad de servicio se convierte en algo totalmente necesario(p.15).

En este mismo sentido (García Buades, 2001) afirma que:

La calidad se ha constituido en uno de los principales objetivos empresariales afectando también a las organizaciones de servicios. Esta relevancia se debe básicamente a los beneficios que la adopción de una estrategia de calidad parece reportar a la organización. Fundamentalmente, la calidad contribuye a mantener y aumentar la cuota de mercado, cada vez más relevante en un entorno altamente competitivo (p.26).

La calidad del servicio, también tiene relación con la fidelidad de los clientes. Si no, imagínese a un huésped o comensal experimentando insatisfacción debido a una pobre calidad de servicio, tenga por seguro que lo más probable es que no regresen jamás. En este mismo sentido (Setó Pamies, 2004) comenta que:

La calidad de servicio se convierte en una de las variables clave para la consecución de la fidelidad del cliente. Aunque ofrece una elevada calidad de servicio no sea suficiente para conseguir una base de clientes fieles, si podemos afirmar que aquellas empresas que lo consigan habrán dado ya, sin duda alguna, un primer paso hacia la fidelidad del cliente (p.153).

En este punto, antes de explicar la relación entre calidad de servicio y satisfacción de los clientes, hay que aclarar que el termino cliente se usa en dos sentidos. El primero para referirse a los clientes que reciben los servicios a los cuales se les denominará clientes externos. En el otro sentido se tiene a los clientes internos, que son los trabajadores de la organización. Según (Dominguez Collins, 2006) afirma con respecto a la categoría de clientes externos que: "Se puede considerar dentro de esta categoría al consumidor final o usuario" (p. 5). Y con respecto al cliente interno Domínguez afirma que: "Son el personal que labora en la empresa es considerado cliente interno, toda vez que es el receptor primario de la misión, de la visión y estrategias formuladas por la organización para lograr la satisfacción del cliente externo" (p.4). 
La calidad tiene una fuerte relación con la satisfacción de los clientes, ya sean estos internos o externos. La satisfacción se dará cuando los clientes perciban que sus expectativas o requerimientos de están cumpliendo. Lo antes mencionado se puede observar cuando se revisan las definiciones del concepto calidad, como por ejemplo la definición que propone (Cuatrecasas, 2010), donde define la calidad como: "El conjunto de características que posee un producto o servicio, así como su capacidad de satisfacción de los requerimientos del usuario" (p.17).

Para entender mejor la relación entre calidad de servicio y satisfacción de los clientes, hay que considerar que la calidad de los servicios puede ser observada desde distintos ángulos. Según (Frydman, 1996) afirma que: "La calidad puede ser vista en función de la competencia, en función absoluta o en función de los parámetros de valor percibido por los clientes (p.221). Esta última visión desde la percepción de los clientes es la más importante, porque ellos son los únicos que saben lo que se necesita para su satisfacción. Esto lo explica (Donelly, 1992) que afirma: "Los clientes tenemos mucho que ofrecer al ámbito directivo. Sabemos lo que se necesita para satisfacer a los clientes, ya que pasamos por la experiencia continua de ser clientes varias veces al día" (p.11).

La relación entre la calidad del servicio y satisfacción de los clientes es meridiana, ambos son aspectos fundamentales a observar con detalle en la administración de empresas turísticas. En este mismo sentido (Setó Pamies, 2004) afirma que: Las primeras investigaciones sobre calidad, centradas en la propia naturaleza de los servicios, intentan proporcionar modelos conceptuales de calidad de servicio, analizando qué es y cómo ésta puede medir- se. Pero, poco a poco, esas investigaciones van evolucionando hacia modelos más complejos en los que el principal objetivo ya no es solo la conceptualización y medición de la calidad de servicio sino también analizar como esta se relaciona con otros conceptos como la satisfacción del cliente y sus intenciones futuras de comportamiento (p.32).

Sin embargo, para otros autores la relación no es tan clara. Según (Hoffman \& Bateson, 2012) afirman que:

Sin lugar a dudas, los dos conceptos de satisfacción del cliente y calidad en el servicio están entrelazados. Sin embargo, la relación entre ambos conceptos no es clara. Algunos consideran que la satisfacción del cliente conduce a la calidad en el servicio percibida, mientras otros creen que la calidad del servicio conduce a la satisfacción del cliente. Además, la relación entre satisfacción del cliente y calidad de servicio y la forma en que estos dos conceptos se relacionan con el comportamiento de compra sigue siendo en gran medida inexplicable (p.319).

Para (Castaño, 2005) la satisfacción es: “Ú1timo elemento del ciclo turístico, además de ser la variable más eficaz en la investigación sobre la calidad de servicio" (p.159).

\section{La Satisfacción laboral}

La satisfacción laboral puede definirse como un estado emocional, que tiene que ver con el bienestar y la felicidad de los trabajadores cuando desempeña sus funciones y perciben un favorable clima donde desarrollarse. Según (Arbaiza Fermini, 2017), explica que la satisfacción laboral tiene dos perspectivas y las explica de la siguiente manera: 
Desde la perspectiva de la organización, la satisfacción laboral es indispensable para atraer y retener a personal talentoso, para lo cual es necesario generar un clima laboral positivo y garantizar condiciones favorables que hagan de la empresa un buen lugar para trabajar. Desde el punto de vista de la persona, la satisfacción laboral tiene beneficios psicológicos, el trabajador encuentra sentido en su trabajo, se siente contento, mejora su calidad de vida y sus relaciones interpersonales. La insatisfacción, por otra parte, afecta su estado de ánimo, su autoestima, su desempeño y puede reflejarse en comportamientos negativos y contraproducentes para la empresa (p.165).

En este mismo sentido las organizaciones deben buscar acciones efectivas para que lotrabajadores se identifiquen con su organización. (Blanchard, Balllard, \& Finch, 2005) afirman que:

Para crear una compañía de manera correcta tiene que ver con los empleados. Todos saben que los empleados son el recurso más importante. Sin embargo, la mayoría de las organizaciones no tienen personas que se identifiquen con la empresa, personas que estén orgullosas de trabajar ahí (p. 112).

Cuando el personal no está identificado con la organización, no se puede esperar su buen rendimiento, ni fidelidad. Según (Leal Millán, De Prado Sagrera, Rodríguez Félix , \& Román Onsalo, 1999) afirman que:

Los estudios relacionados a la satisfacción laboral son de innegable importancia. Además, afirma que: Uno de los síntomas de mal funcionamiento de la organización es la baja satisfacción de sus empleados. Son muchos y muy importantes los aspectos relacionados con la satisfacción laboral: rotación, absentismo, rendimiento, estrés, conflictos etc. Teniendo en cuenta los altos costes de estas variables, parece deseable una alta satisfacción laboral, ya que se relaciona con resultados organizacionales positivos (p.116).

Para que exista la satisfacción en los trabajadores, ellos deben percibir que la organización les brinda un buen clima laboral. Según (Chiavenato, 2011) afirma que: "cuando el clima organizacional sube, este se traduce en relaciones de satisfacción, animo, interés, colaboración etc. (p. 49). Sin embargo, no hay que olvidarse de brindar a los trabajadores el salario emocional, que es un factor determinante para obtener un buen clima laboral.

Entonces es importante que las organizaciones busquen con esmero la satisfacción de los trabajadores. Según (Baguer Alcalá, 2008) afirman que: "Los directivos deben procurar dotar a las organizaciones de unas condiciones de trabajo que conduzcan a la máxima satisfacción de personas" (p.171). Y ellos, seguramente responderán con reciprocidad. En este mismo sentido (Chang \& Kelly, 1994) afirman que: "Para obtener y conservar la lealtad del cliente mediante un proyecto de satisfacción del cliente es preciso comenzar por el interior de la organización" (pág. 5).

Es decir, hay que comenzar a satisfacer a los trabajadores. Según (Hellriegel, Jackson, \& Slocum, 2010) "existen cuatro factores que sirven para explicar la motivación y la satisfacción de los empleados. Estos factores son: 
El comportamiento de los administradores, el diseño de puesto, el contexto organizacional y las diferencias individuales" (p.23), que son dimensiones de la satisfacción laboral.

\section{Calidad de vida laboral y satisfacción de los empleados}

El hotel Marriot, considera como pieza clave del éxito, a sus trabajadores, situación ideal, pero que no es compartida por todos. La realidad es que se observan más casos donde el pensamiento es a la inversa, donde se desprecia y maltrata a los trabajadores encargados del servicio, esto naturalmente generará insatisfacción en ellos. En este mismo sentido J. W. Marriot Jr. Como se citó en (Albrecht k., 1997) afirma que:

La idea del servicio, o la de desempeñar una labor de servicio, se considera como degradante y significa un status bajo. Muchos ejecutivos y gerentes consideran a los trabajadores de servicios como gente sin importancia y fácilmente reemplazables. Pero en realidad la gente que se ocupa de los servicios es la más importante en la organización. Sin ellos no hay producto, venta ni ganancia (s/n).

(Goeldner \& Brent Ritchie, 2011), donde se comenta lo siguiente:

Marriot es conocido en toda la industria por su forma práctica de administrar y ha construido una cultura altamente reconocida que enfatiza la importancia de su gente y reconoce el valor que ésta le da a la organización. La cultura con espíritu de servicio Marriot International, se basa en la filosofía de negocios creada hace ochenta años por los padres de Marriot, J. Willard y Alice S. Marriot. Ellos decían, cuiden a sus asociados y éstos cuidarán a sus huéspedes (p.182).

Entonces el factor humano sin duda es un factor determinante para que las organizaciones de servicios brinden calidad. Por lo tanto, no deberán descuidar la calidad de vida laboral de sus empleados. En este mismo sentido (Chiavenato, 2012) afirma que:

Si la calidad de vida laboral es pobre, conducirá a la alienación del empleado y a la insatisfacción, a mala voluntad, a la reducción de la productividad, a comportamientos contraproducentes (ausentismo, rotación, robo, sabotaje, militancia sindical, etc.), si la calidad de vida laboral es buena, generará un clima de confianza y respeto mutuo en que el individuo tenderá a aumentar sus contribuciones y elevar sus oportunidades de éxito psicológico, y la administración tenderá a reducir mecanismos rígidos de control (p.408).

Comoverán, lacalidaddevidalaboralescrucial, si se quiere lograr la calidad del servicio. Bajos niveles de calidad de vida laboral, generan trabajadores insatisfechos y esto tendrá un reflejo en detrimento de la satisfacción de los clientes. (Barlow \& Moller, 2001), afirman que: "Los clientes insatisfechos hablarán a unas ocho o diez personas del mal servicio que han recibido. Una de cada cinco personas se lo dirá a veinte personas" (p. 47).

\section{Población y muestra de la investigación}

Para calcular población y muestra, se trabajo considerando un universo 3391 clientes. Para delimitar la población, se dividió 3391 entre cuatro semanas, lo que dio un total por semana de 847 clientes. Para calcular la muestra se ha considerado que la población que va a ser encuestada tiene necesidades y característi- 
cas similares, en vista de tal homogeneidad, se utilizó un grado de confianza al $0.95 \%$

$$
\begin{array}{lll}
\text { - } & \text { Error muestral (E). } & 0.5 \\
\text { - } & \text { Proporción (p) } & 0.5 \\
\text { - } & \text { Proporción (q } & 0.5 \\
\text { - } & \text { Grado de confianza(a) } 0.95 \\
\text { - } & \text { Valor de (Z) } & 1,645 \\
\text { - } & \text { Población (N) } & 847 \\
\text { - } & \text { La muestra (n) } & 68
\end{array}
$$

Según la fórmula del muestreo, esta arrojó una cifra de 68, que será el número de encuestas a realizar a los clientes del Club Departamental Arequipa. La muestra se consideró probabilística aleatoria simple, porque cualquier cliente tiene la misma oportunidad de ser elegido sin que intervengan, en absoluto, la intención o los propósitos del investigador. Esta selección se realizó completamente al azar. En el caso de la población de trabajadores del club, no se calculó muestra, sino más bien, se realizó un censo, debido a la moderada cantidad de trabajadores dedicados a la atención al cliente, que suman un total de 12 colaboradores.

\section{RESULTADOS Y DISCUSIÓN}

En la figura 1, se puede observar que el nivel de satisfacción de los trabajadores, antes de la aplicación del plan de calidad de vida laboral obtuvo una puntuación de 3.58 lo que se consideró moderadamente satisfecho.

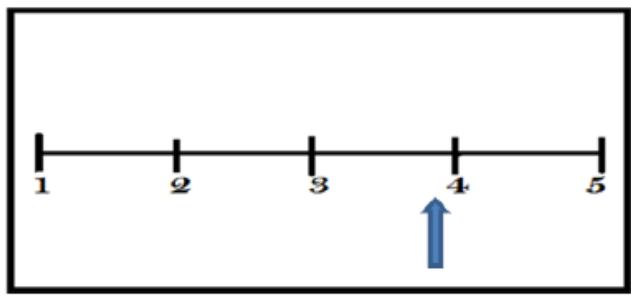

Fuente. Elaboración propia, 2018

Figura 1. Niveles de satisfacción de los trabajadores del Club Departamental Arequipa, antes de la aplicación del plan de calidad de vida laboral.
En la figura 2, se puede observar que, tras la aplicación del plan de calidad de vida laboral, se incrementó los niveles de satisfacción laboral de los trabajadores del club, llegando a una puntuación de 3.93.

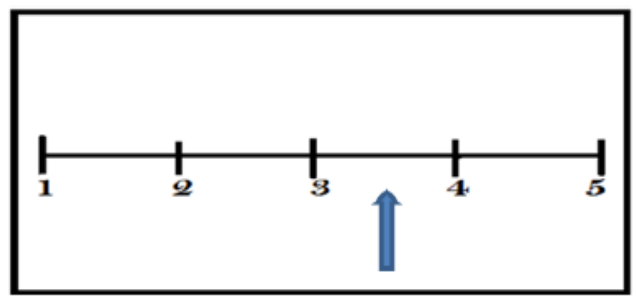

Fuente. Elaboración propia, 2018.

Figura 2. Niveles de satisfacción de los trabajadores del Club Departamental Arequipa, después de la aplicación del plan de calidad de vida laboral.

En la figura 3, se puede observar los niveles de satisfacción de los clientes, respecto a la calidad del servicio, antes de la aplicación del plan de calidad de vida laboral. Donde se llegó a una puntuación de 4.127, lo que se puede interpretar como clientes satisfechos.

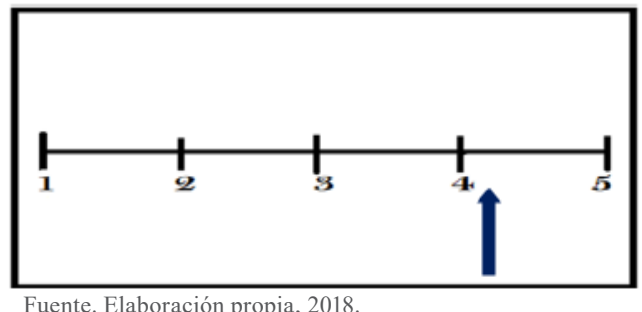

Figura 3. Niveles de satisfacción de los clientes del Club Departamental Arequipa, antes de la aplicación del plan de calidad de vida laboral.

En la figura 4, se observa que tras la aplicación del plan de calidad de vida laboral y el incremento de la satisfacción laboral, también aumento concomitantemente los niveles de satisfacción del cliente, llegando a una puntuación de 4.35 lo que se sigue interpretando como clientes satisfecho, pero en mayor medida. 


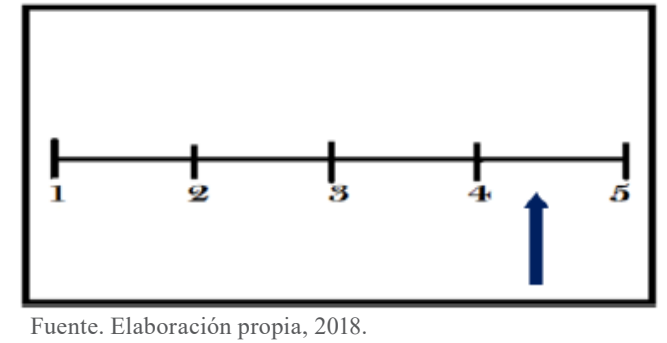

Figura 4. Niveles de satisfacción de los trabajadores del Club Departamental Arequipa, después de la aplicación del plan de calidad de vida laboral.
Con respecto a la correlación se puede observar en la tabla 1 , los resultados del análisis estadístico. Se utilizó el coeficiente de Pearson, donde en cada uno de los factores de la satisfacción laboral, se observó un P-valor menor al valor de alfa. Lo que significa que existe correlación.

Tabla 1. Resumen de los resultados del análisis de correlación de Pearson

\begin{tabular}{l|l|l}
\hline $\begin{array}{l}\text { Correlación de Pearson } \\
\text { P=0,000 (comportamiento de los }\end{array}$ & $<$ & $\alpha=0,05$ \\
administradores) & & \\
\hline $\mathrm{P}=0,000$ (contexto organizacional) & $<$ & $\alpha=0,05$ \\
\hline $\mathrm{P}=0,000$ (diseño de puestos) & $<$ & $\alpha=0,05$ \\
\hline $\mathrm{P}=0,000$ (diferencias individuales) & $<$ & $\alpha=0,05$ \\
\hline $\mathrm{P}=0,000$ (satisfacción laboral) & $<$ & $\alpha=0,05$ \\
\hline
\end{tabular}

Fuente. Elaboración propia, 2018.

Conclusión: En todos los casos el P-Valor es menor que el valor de alfa, por lo tanto, hay evidencia estadística para afirmar que la satisfacción laboral está relacionada significativamente con la satisfacción del cliente.

En la tabla 2, se puede observar el valor de la $\mathrm{R}$ de Pearson, que es de 0,507 lo que se interpreta como correlación positiva media. Esto quiere decir que existe relación entre los niveles de satisfacción laboral y los niveles de satisfacción de los clientes, de tal manera que ha mayor satisfacción laboral, habrá mayor satisfacción de los clientes. 
Tabla 2. Valor de la R de Pearson

\begin{tabular}{|c|c|c|c|}
\hline & & $\begin{array}{l}\text { SATISFACCIÓN } \\
\text { LABORAL }\end{array}$ & $\begin{array}{l}\text { SATISFACCIÓN DEL } \\
\text { CLIENTE }\end{array}$ \\
\hline \multirow{5}{*}{$\begin{array}{l}\text { SATISFACCIÓN } \\
\text { LABORAL }\end{array}$} & Correlación de Pearson & 1 & 507 \\
\hline & Sig. (bilateral) & & 000 \\
\hline & $\begin{array}{l}\text { Suma de cuadrados y productos } \\
\text { cruzados }\end{array}$ & 1,866 & 308 \\
\hline & Covarianza & 098 & 016 \\
\hline & N & 12 & 12 \\
\hline \multirow{5}{*}{$\begin{array}{l}\text { SATISFACCIÓN DEL } \\
\text { CLIENTE }\end{array}$} & Correlación de Pearson & 507 & 1 \\
\hline & Sig. (bilateral) & 000 & \\
\hline & $\begin{array}{l}\text { Suma de cuadrados y productos } \\
\text { cruzados }\end{array}$ & 308 & 507 \\
\hline & Covarianza & 016 & 016 \\
\hline & N & 68 & 68 \\
\hline
\end{tabular}

Fuente. Elaboración propia, 2018.

En la tabla 3, se puede observar los resultados de la prueba Anova que, en ambos grupos, tanto como de trabajadores como de clientes, arrojó un P-valor menor al valor de alfa. Lo que significa que se rechaza la hipótesis nula y se acepta la hipótesis de investigación, que propone una diferencia significativa entre medias estadísticas.

Tabla 3. Resultado del análiwsis de la prueba de homogeneidad de varianzas con Anova

$$
\text { Prueba Anova }
$$

\begin{tabular}{l|l|l}
\hline$P=0,000$ & $<$ & $\alpha=0,05$ \\
& & \\
\hline
\end{tabular}

Conclusión: El P-Valor es menor que el valor de alfa. Por lo tanto, existe suficiente evidencia estadística para rechazar la hipótesis nula. 
La satisfacción laboral y la satisfacción del cliente en la atención brindada

en el Club Departamental Arequipa

\section{CONCLUSIONES}

Según (Kotler, Bowen, Makens, Moreno, \& Reina P, 2005) "La satisfacción de los empleados y del cliente están directamente relacionadas" (p.210), y los resultados de esta investigación así lo demuestran. Por lo tanto, si los trabajadores tienen sentimientos de alegría, felicidad y beneplácito; estos sentimientos positivos serán el motor que mueva a los empleados, y esto se verá reflejado positivamente en ellos, ya sea atendiendo al cliente o produciendo para él. Por lo tanto, como parte de la estrategia empresarial del Club Departamental Arequipa, siempre deberán estar presentes los programas de calidad de vida laboral, que se sabe incrementan concomitantemente la satisfacción de los empleados y de sus clientes.

\section{REFERENCIAS BIBLIOGRÁFICAS}

Acerenza, M. (2004). Fundamentos del Marketing Turístico. México: Editorial Trillas S.A.

Albrecht, k. (1997). La revolución del servicio: Lo únnico que cuenta es un cliente satisfecho. Bogotá: 3R EDITORES.

Arbaiza Fermini, L. (2017). Liderazgo y Comportamiento Organizacional. Lima: Universidad ESAN.

Arbaiza Fermini, L. (2014). Administración y Organización. Lima: ESAN.

Baguer Alcalá, A. (2008). Dirección de personas: Un timón en la tormenta. Madrid: Diaz de Santos.

Barlow, J., \& Moller, C. (2001). Una Queja es un Regalo: Como utilizar la opinión de los clientes para las mejoras continuas. Barcelona: Berret-Koehler Publishers.
Blanchard, k., Balllard, J., \& Finch, F. (2005).

Clientemanía: Nunca es demasiado tarde para construir una compañia centrada en el cliente. Bogotá: Grupo Editorial Norma.

Castaño, J. (2005). Psicología Social de los Viajes y del Turismo. Madrid: Thompson Editores.

Chang, R. Y., \& Kelly, P. (1994). Satisfacer primero a los clientes internos. Buenos Aires: Granica S.A.

Chiavenato, I. (2011). Administración de recursos humanos: El capital humano de las organizaciones. México: Mc Graw Hill.

Chiavenato, I. (2011). Introducción a la teoría general de la administración. México: Mc Graw Hill.

Chiavenato, I. (2012). Gestión del talento humano. México: Mc Graw Hill.

Cobra, M. (2000). Marketing de Servicios. Bogotá: Mc Graw Hill Interamericana, S.A.

Couillaud, D. (2006). Marketing Turístico y Hotelero. Lima: FCCTYP-USMP.

Cuatrecasas, L. (2010). Gestión Integral de la Calidad. Barcelona: Profit Editorial.

Dominguez Collins, H. (2006). El servicio invisible. Bogotá: ECOE.

Donelly, J. H. (1992). Cómo conquistar al cliente. Barcelona: Paidós Ibérica.

Elias, J. (2000). Clientes contentos de verdad: 
La satisfacción laboral y la satisfacción del cliente en la atención brindada en el Club Departamental Arequipa

clave para comprender a clientes y ususarios. Barcelona: Gestión .

Fernández , P., \& Bajac, H. (2012). La gestión de marketing de servicios: principios y aplicaciones para la actividad gerencial. Buenos Aires: Granica.

Frydman, A. (1996). La esencia del marketing de servicios. Buenos Aires: EDICIONES MACCHI.

García Buades, E. (2001). Calidad de servicio en hoteles de sol y playa. Madrid: Editorial Síntesis.

Gasalla, J. M. (2009). La nueva dirección de personas. Madrid: PIRAMIDE.

Goeldner, C. R., \& Brent Ritchie, J. R. (2011). Turismo: Planeación, administración y perspectiva. México: Limusa Wiley.

Grande Esteban, I. (2005). Marketing de los servicios. Madrid : ESIC.

Hellriegel, D., Jackson, S., \& Slocum, J. (2010). Administración: un enfoque basado en competencias. México: Cengage Learning.

Hoffman, K. D., \& Bateson, J. E. (2012). Marketing de Servicios. México: Cengage Learning Editores S.A.

Kotler, P., \& Armstrong, G. (2008). Fundamentos del Marketing. México: Pearson Educación.

Kotler, P., Bowen, J., Makens, J., Moreno, R. R., \& Reina P, M. D. (2005). Marketing para Turismo. Madrid: PEARSON EDUCACIÓN.
Leal Millán, A., De Prado Sagrera, A., Rodríguez Félix , \& Román Onsalo, M. (1999). El factor humano en las relaciones laborales. Madrid: PIRAMIDE.

Martin, W. (1992). Dirección de los Servicios de Calidad al Cliente: Guía práctica para operaciones de servicio. México: Iberoaméricana S.A.

Olvera Romero, I. (2013). El cliente y la calidad en el servicio. México: Trillas.

Parra López, E., \& Calero García, F. (2006). Gestión y Dirección de Empresas Turísticas. Madrid: Mc Graw Hill.

Planells, M., \& Montserrat, C. (2012). Servicios de Información Turística. Madrid: EDITORIA L SINTESIS, S.A.

Setó Pamies, D. (2004). De la calidad de servicio a la fidelidad del cliente. Madrid: ESIC.

Zeithaml, V., Parasuraman, A., \& Berry, L. (1993). Calidad Total en la Gestión de Servicios. Madrid: Díaz de Santos S.A. 